\title{
Healthy Eating: How Do We Define It and Measure It? What's the Evidence?
}

\author{
Christy C. Tangney, PhD, CNS, \\ Beth A. Staffileno, PhD, RN, \& \\ Heather E. Rasmussen, PhD, RD
}

\begin{abstract}
Christy C. Tangney, PhD, CNS, FACN is a Professor with the Department of Clinical Nutrition at Rush University Medical Center in Chicago, IL, and can be reached at ctangney@rush.edu. Beth A. Staffileno, PhD, RN is an Associate Professor with the Department of Adult Health and Gerontological Nursing at Rush University Medical Center.

Heather E. Rasmussen, PhD, RD is an Associate Professor with the Departments of Clinical Nutrition at Rush University Medical Center.
\end{abstract}

\begin{abstract}
A healthy diet can be defined in many ways, including defining one's food intake by a dietary pattern. As described in the Dietary Guidelines for Americans Committee report, there are several defined dietary patterns associated with lower rates of chronic diseases. These include the Healthy Eating Index, Dietary Approach to Stop Hypertension, and those based on the Mediterranean diet. This review will focus on guiding health care professionals, including nurse practitioners, how a healthy diet pattern is defined, how it is measured, and a summary of recent evidence supporting the healthfulness of these dietary patterns.
\end{abstract}

Keywords: accordance, Alternate Healthy Eating Index, dietary patterns, Healthy Eating Index, Mediterranean 
Lealthy eating can be defined in many ways to accommodate different tastes and cultures. One resource many nutritionists rely on to define healthy eating is the Dietary Guidelines for Americans (DGA). The DGA was established by congressional mandate under the 1990 National Nutrition Monitoring and Related Research Act (Public Law 101-445) and is issued every 5 years jointly by the United States Department of Agriculture (USDA) and the Department of Health and Human Services. These departments call on a group of external food and nutrition experts who comprise the Dietary Guidelines Advisory Committee (DGAC). Experts examine and evaluate all available research (augmented by the systematic reviews of the nutrition and health literature) and make recommendations that are summarized in the DGAC ScientificReport. ${ }^{1}$ The report is open to public comment and then is further reviewed, summarized, and released as the DGA by the department secretaries. ${ }^{2}$ The process is designed to be transparent and unbiased. The aims of the DGA are to translate this information into practical food-based recommendations that promote overall health and inform federal policy and programs such as the Older Americans Act Nutrition Services Program and the Supplemental Nutrition Assistance Program, which impact millions of persons each day. ${ }^{3}$ The 2010 DGAC was the first to emphasize healthy dietary patterns as opposed to the prior reports that focused on recommendations for individual dietary components or nutrients.

Although the 2005 DGAC did describe the Dietary Approach to Stop Hypertension (DASH) food pattern, an even greater emphasis was placed on food groups and nutrients that make up several healthy diet patterns in 2010. Moreover, the DGAC detailed recommendations that focused on behaviors contributing to these patterns including accentuating fruit, vegetable, whole grain, and seafood consumption with admonitions regarding how snacking, fast food, and breakfast habits have changed over the past 3 decades. This theme is further emphasized in the 2015-2020 DGAC (which henceforth will be referred to as the 2015 DGAC).

One universal message nutritionists want all health professionals including nurse practitioners to promote is the adoption of overall healthy dietary patterns, for which there can be many. This approach would be more helpful and healthful than simply advocating a superfood or restriction of a particular bad food or dietary component. People do not eat nutrients but rather consume foods or meals comprised of combinations of foods and beverages that influence what and how well nutrients are absorbed, metabolized, or stored. ${ }^{4}$ Dietary patterns reflect a combination of food groups, food items, and/or beverages consumed with specified 
habitual frequencies. The identification of optimal dietary patterns is often derived from large-scale population studies in which usual diet is described with broad strokes because the dietary assessment method most often used is the food frequency questionnaire (FFQ). This tool consists of a long list of food items to capture a variety of food choices and often with defined portion and frequencies (per day, per month, and so on) that the respondent completes.

\section{A priori dietary patterns}

There are, in fact, 3 approaches in which dietary patterns can be derived. ${ }^{5}$ The first includes the a priori dietary patterns, of which 3 common patterns are shown in the first 3 columns in Table 1. These 3 a priori patterns include 1) the Healthy US Pattern, which can be quantified with either the Healthy Eating Index (HEI) 20106,7 or an alternative version of this index, the Alternate Healthy Index [AHEI] 2010; 2) the Healthy Mediterranean-style Pattern as exemplified by the MedDietScore, ${ }^{8}$ the Mediterranean Diet Score, ${ }^{9}$ or the alternate Mediterranean score (aMed) ${ }^{10}$; and 3) the DASH score. ${ }^{11,12}$ There are many versions and modifications of these indices, which can add confusion when relating dietary patterns to clinical outcomes. We recently reviewed the different scoring algorithms for the DASH diet; 13 different DASH scoring tools were identified. ${ }^{13}$ There are also many ways to score a Mediterranean-like dietary pattern in the literature. ${ }^{14,15}$

\section{A posteriori method}

The second way to define a dietary pattern is known as the data-driven or a posteriori method whereby all the different foods the participants report eating are statistically reduced in number by grouping foods together to become a recognizable pattern of consumption. ${ }^{16}$ The datadriven patterns may be derived by a variety of statistical procedures. Factor and cluster analytical approaches are common; in both, patterns/ clusters are labeled or named by the researchers. Common examples of such patterns include the Prudent or its counterpart Westernized diet patterns. Two other data-driven approaches include reduced rank regression along with classification and regression tree (CART) analysis. ${ }^{17}$ In these latter 2 procedures, one is assessing what foods describe the most variation in an outcome (i.e., systolic blood pressure, incident stroke, or cognitive decline). Because a posteriori-identified patterns are specific to the 
population sample in which the analyses are being performed, they are often not applicable to other groups of individuals. None of these patterns are depicted in Table 1.

\section{Qualitative dietary patterns based on food and beverage preferences}

Finally, a third approach is based on a description of food and beverage preferences or what foods are excluded or selected routinely. The method is more qualitative and relies on self-reported behaviors by the individual. Examples are vegetarian or 1 of its many variations (i.e., ovolacto vegetarian, vegan, and so on). A healthy vegetarian dietary pattern has been developed by the 2015 DGAC and relies largely on the reported intake patterns of self-identified vegetarians in National Health and Nutrition Examination Survey. ${ }^{1}$ This healthy vegetarian pattern is presented in the right-most column in Table 1.

\section{Healthy eating patterns}

The DGAC 2015 chose to highlight several a priori (and 1 qualitative one, the vegetarian) dietary patterns (Table 1) that have been described by many research groups in relation to health benefits. A growing body of evidence attests to the reduced risk of diet-related chronic diseases, such as cardiovascular diseases, diabetes, and cancer. The Healthy US Pattern specified in Table 1 can be graphically translated into its simplest form as Choose MyPlate ( http://www.choosemyplate.gov/tools-supertracker ), and, thus, foods or beverages to limit are not part of this graphic. The US pattern also can be quantified in terms of a scoring algorithm known as HEI 2010 (Table 2). HEI 2010 reflects the third revision of a pattern that conforms to federal dietary recommendations. The original HEl contained 10 components with an optimal score of 100 , but no energy adjustment was applied..$^{18}$ Unlike the earliest version, the later revisions (HEl 2005 and HEI 2010) are comprised of 12 components. Each is scored by comparing with a target amount of food or nutrient to be consumed each day relative to energy intake rather than absolute amounts. ${ }^{6,7,19}$ In this scoring pattern, the maximum number (100 points) reflects an optimal pattern. Both foods to be included and foods to be limited comprise this pattern. Fruits (emphasis on whole), vegetables with specified targets for dark green and orange vegetables, whole grains, dairy, and then seafood and plant proteins are recommended. One receives higher point values 
the closer the intake amounts equate to the target number of servings. Limits are established for refined grains, sodium, and the proportion of energy from empty calories (i.e., solid fats, alcohol, and added sugars), and these are reverse scored; thus, lower amounts consumed each day would be assigned a proportionally higher point value. An update to $\mathrm{HEI} 2010$ is anticipated because although the 2015 DGAC recommends specific target servings for nuts (Table 1), there is no stand-alone component for nuts in HEI 2010. (It is currently incorporated in the seafood and plant protein component.) Another possible change for the next $\mathrm{HEI}$ version is a limit on the number of servings of red and processed meats, which is consistent with the 2015 DGAC guideline report.

Table 1. Healthy Dietary Patterns in the 2015-2020 Dietary Guidelines Advisory Committee Report

\begin{tabular}{|c|c|c|c|c|}
\hline Component & $\begin{array}{l}\text { Healthy } \\
\text { US } \\
\text { Pattern }\end{array}$ & $\begin{array}{c}\text { Healthy } \\
\text { Mediterranean-style } \\
\text { Pattern }\end{array}$ & DASH & $\begin{array}{l}\text { Healthy } \\
\text { Vegetarian } \\
\text { Pattern }\end{array}$ \\
\hline Total fruit (cups) ${ }^{a}$ & 2 & 2.5 & 4 & 2 \\
\hline Whole fruit (not juice) & - & - & - & - \\
\hline Total vegetables (cups) ${ }^{a, b}$ & 2.5 & 2.5 & 4 & 2.5 \\
\hline Dark greens & $1.5 / \mathrm{wk}$ & $1.5 / \mathrm{wk}$ & - & $1.5 / \mathrm{wk}$ \\
\hline Red/orange & $5.5 / \mathrm{wk}$ & $5.5 / w k$ & - & $5.5 / \mathrm{wk}$ \\
\hline Starchy & 5/wk & 5/wk & - & 5/wk \\
\hline Legumes & $1.5 / \mathrm{wk}$ & $1.5 / \mathrm{wk}$ & $4-5 / w^{c}$ & $3 / w k$ \\
\hline Total grains (oz equivalent)a & 6 & 6 & 6 & 6.5 \\
\hline Whole grains & 3 & 3 & 3 & 3.5 \\
\hline Refined grains & 3 & 3 & 3 & 3 \\
\hline Dairy (cups) ${ }^{\mathrm{a}}$ & 3 & 2 & 3 & 3 \\
\hline Proteins (oz equivalent)a & 5.5 & 6.5 & - & 3.5 \\
\hline Nuts/seeds & 4/wk & 4/wk & $4-5 / w k$ & $7 /$ wk \\
\hline Red and processed meats & $12.5 / w k$ & $12.5 / w k$ & $\leq 6 / \mathrm{wk}$ & - \\
\hline Poultry & $10.5 / w k$ & $10.5 / w k$ & - & - \\
\hline Seafood & 8/wk & 15/wk & - & - \\
\hline Eggs & $3 / w k$ & $3 / w k$ & - & $3 / w k$ \\
\hline Processed soy (tofu) & $0.5 / w k$ & $0.5 / \mathrm{wk}$ & - & 8/wk \\
\hline \multicolumn{5}{|l|}{ Fats $^{a}$} \\
\hline Solid fats, g (tsp) & $18(2)$ & $17(0.9)$ & $2-3$ & $21(2.3)$ \\
\hline Oils, g (tsp) & $27(3)$ & $27(3)$ & - & $27(3)$ \\
\hline Sweets, added sugars, g (tsp)a & $30(7.5)$ & $29(7.25)$ & - & $36(9)$ \\
\hline Sugar-sweetened beverages/fruit juice & - & & $\leq 5 / w k$ & - \\
\hline
\end{tabular}

DASH = Dietary Approach to Stop Hypertension; $g$ = grams.

a. Values are expressed as amount per day and are boldfaced. Scoring standards are based on cup and ounce equivalents, where $1 \mathrm{oz}=28.3 \mathrm{~g}$ and $1 \mathrm{cup}=225 \mathrm{~mL}$.

b. Other vegetables and starchy vegetables are not shown here but contribute to total vegetables.

c. The total amount, includes the amount counted toward protein foods. 
Another well-known variant of the US Healthy Pattern is the Harvard Healthy Eating Plate ( http://www.hsph.harvard.edu/nutritionsource/ healthy-eating-plate ) and is defined or quantified using the AHEI as shown in Table 2. McCullough et al ${ }^{20}$ developed the original $\mathrm{AHEl}$, which was updated to the AHEI 2010. ${ }^{21}$ Although modeled after the $\mathrm{HEl}$, the AHEl was further modified to include food items and nutrients associated with a lower risk of chronic diseases based on numerous reports from the Nurses' Health Study and the Health Professionals Study participants. The restriction of red and/or processed meat intake is reflected in how this key component is scored, as is that of 2 other components: 1) sugar-sweetened beverage and fruit juice consumption and 2) trans fat intake. Note these restrictions, although indirectly presented in the Healthy US Pattern, have not been operationalized into scoring components of the HEI 2010. Also note that dairy is not a component of the AHEl 2010, in contrast to many other patterns. The AHEl consists of 11 components and a total possible score of 110 points (Table 2).

The DASH dietary pattern has been studied for many years in both controlled feeding trials ${ }^{11,22}$ and free-living trials. ${ }^{23,24}$ The first 2 randomized feeding trials were conducted to assess the efficacy of this pattern in terms of blood pressure lowering. Women whose diets reflect strong accordance to 1 version of the DASH pattern ${ }^{12}$ had fewer strokes and less coronary heart disease during 24 years of follow-up. How this pattern is scored also has had many interpretations. Nevertheless, a much greater emphasis on fruit and vegetable servings in DASH is evident when dietary patterns are compared, as shown in Table 1. In concert with the Healthy US Pattern, DASH recommends fruits and vegetables daily, legumes, lowfat dairy, nuts and seeds, and limited meats. Although the DASH diet does not specifically recommend a sodium limit, DASH and the restriction of dietary sodium have been shown to be effective at blood pressure lowering. ${ }^{22}$ Thus, a sodium cutoff is often a component of this pattern. Dietary sodium restriction is also an important component of both the HEI 2010 and AHEI 2010 scoring algorithms (Table 2).

In contrast, dietary sodium restriction is rarely mentioned for either the vegetarian or Mediterranean-style dietary patterns. One of the first Mediterranean diet pattern scores was developed in the 1960s based on the observed association with greater survival in the people of Greece. In comparison with previously mentioned patterns, in the Mediterranean-style pattern, ${ }^{9}$ emphasis is placed on equivalent amounts of fruits and vegetables, less dairy, and greater frequency of seafood/fish consumption. A further modification, the aMed score, was created for use 


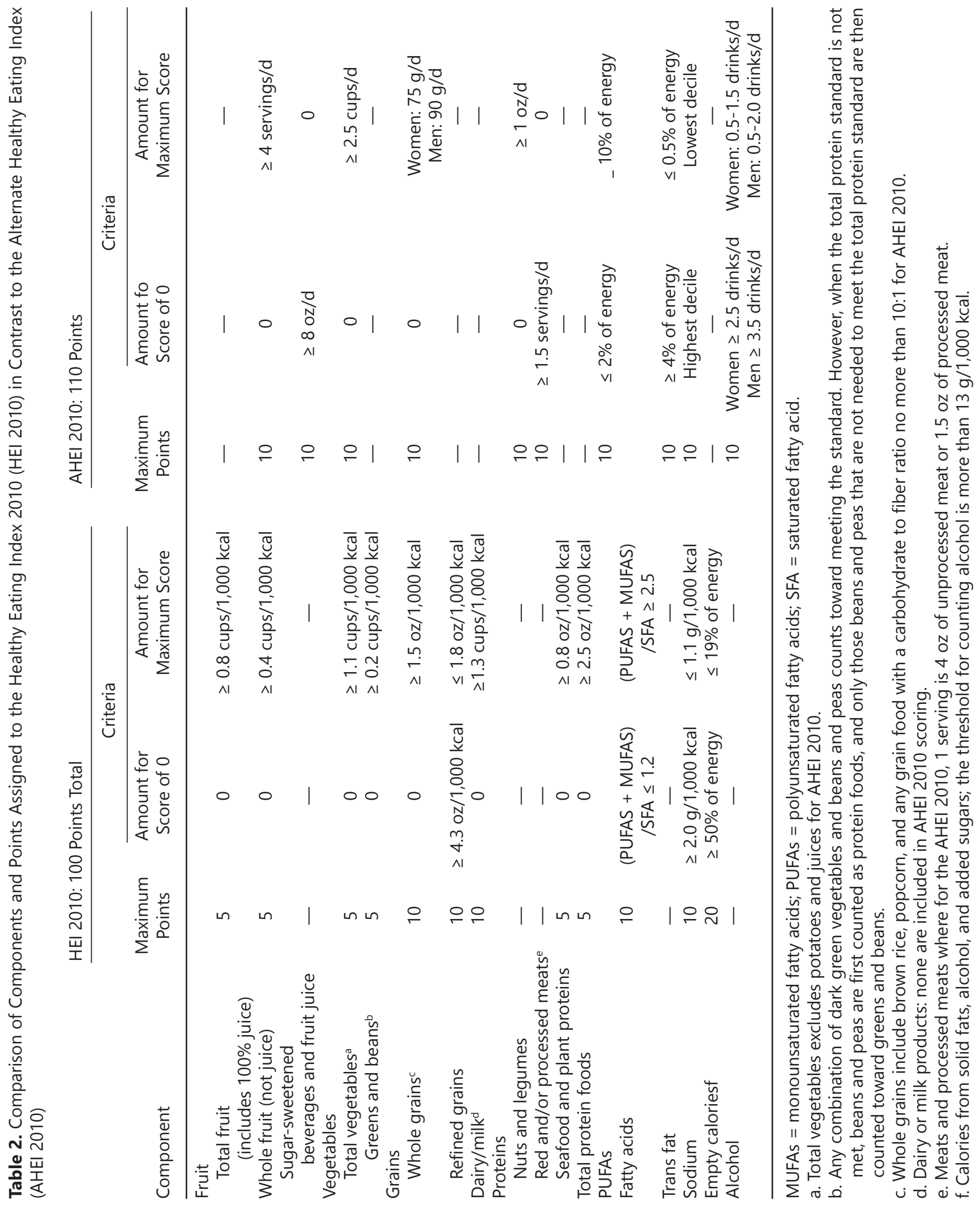


in American populations..$^{25}$ Like the common European score, ${ }^{9}$ it has 9 components for a total of 9 points, where 1 point is assigned for intakes at or greater than the sex-specific median for each of the healthy food components such as whole grains, fruits, nuts, legumes, and so on in the population sample being studied (Table 3). In addition to the aMed scoring tool, there is another Mediterranean score with 11 components, each with a maximum score of $5 .^{8}$ The latter scoring approach does not depend on sex-specific median intakes, but rather scores are assigned in relation to the target number of servings of each food component. We also include a screener, known as the Mediterranean Eating Pattern for Americans II (MEPA II), in the right-most column of Table 3.

\section{Assessing accordance and adherence to dietary patterns}

The first step in measuring accordance to any of these healthy dietary patterns is an assessment of one's diet through single or multiple 24hour recalls or FFQs. These can be self-administered or completed by a skilled interviewer. They can be completed on paper or electronically. Recently, the federal government (the National Cancer Institute) has developed and released a web-based system known as the Automated SelfAdministered 24-hour Dietary Recall System (http://epi.grants.cancer.gov/ asa24/), which is free to researchers, clinicians, and teachers. The mode of administration depends on the setting and/ or the number of participants to be assessed, costs, and whether acute (24-hour recall) or usual/ chronic (FFQ) intake patterns are desired.

The next step involves categorization of the intake frequencies of nutrients, foods, or beverages into either ordinal or dichotomous scores. For most dichotomous scores (i.e., aMED), cutoffs must be assigned based on sex-specific median intakes of the key food or nutrient components. In contrast, for the HEl 2010 food component scores, both minimum and maximum scores are assigned based on target servings per 1,000 kcal; for other index scoring such as the MedDietScore, incremental target servings are assigned point values from 1 to 5 . At this point in time and to the best of our knowledge, there is no smartphone application that generates total and individual HEl 2010 component scores once foods or beverages have been recorded throughout the day. As previously mentioned, there is the web-based Automated Self-Administered 24-hour Dietary Recall System available to all, but analyses will be instantaneous. On the other hand, the food tracker component of Supertracker developed by the Center for Nutrition Promotion and Policy at the USDA will 


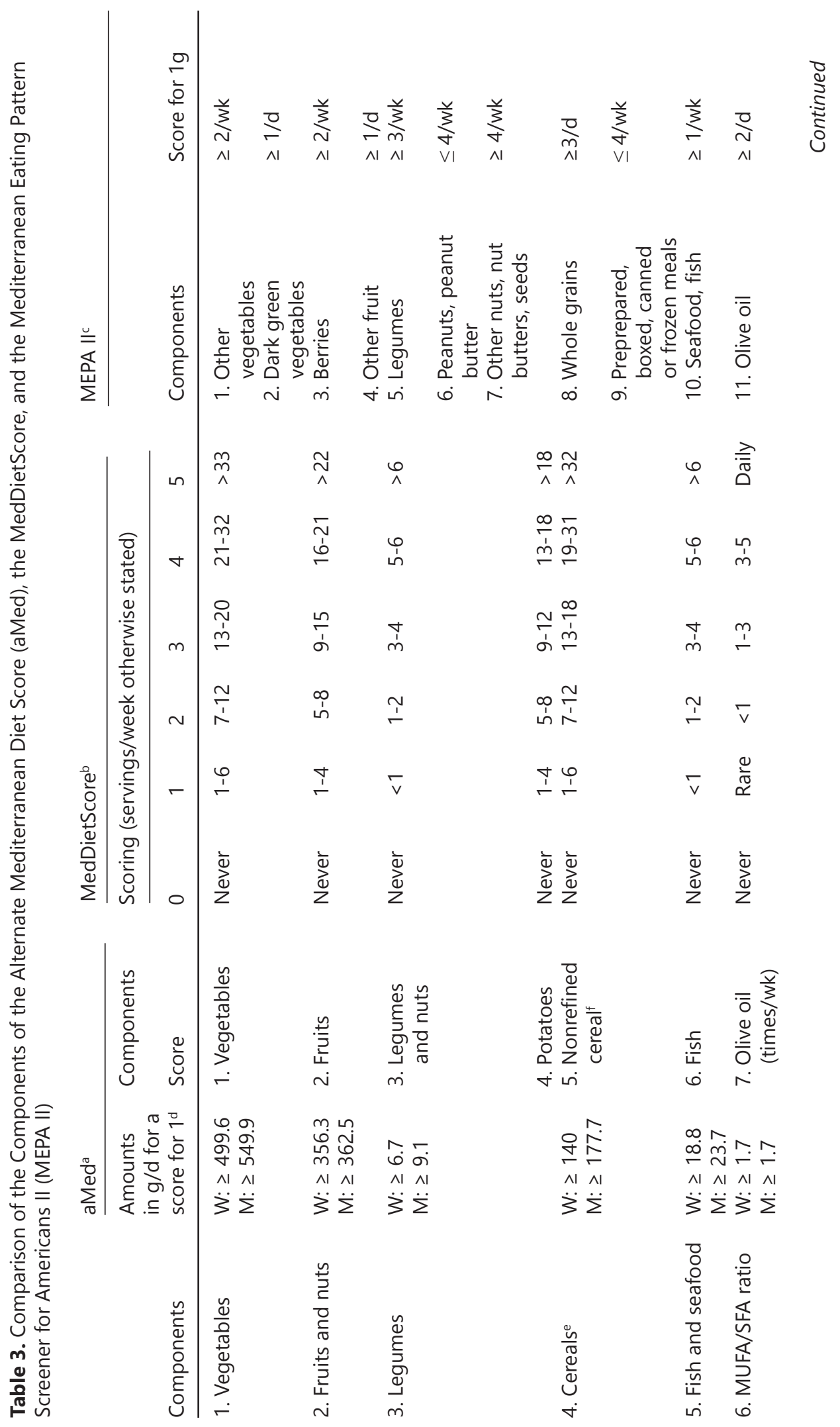




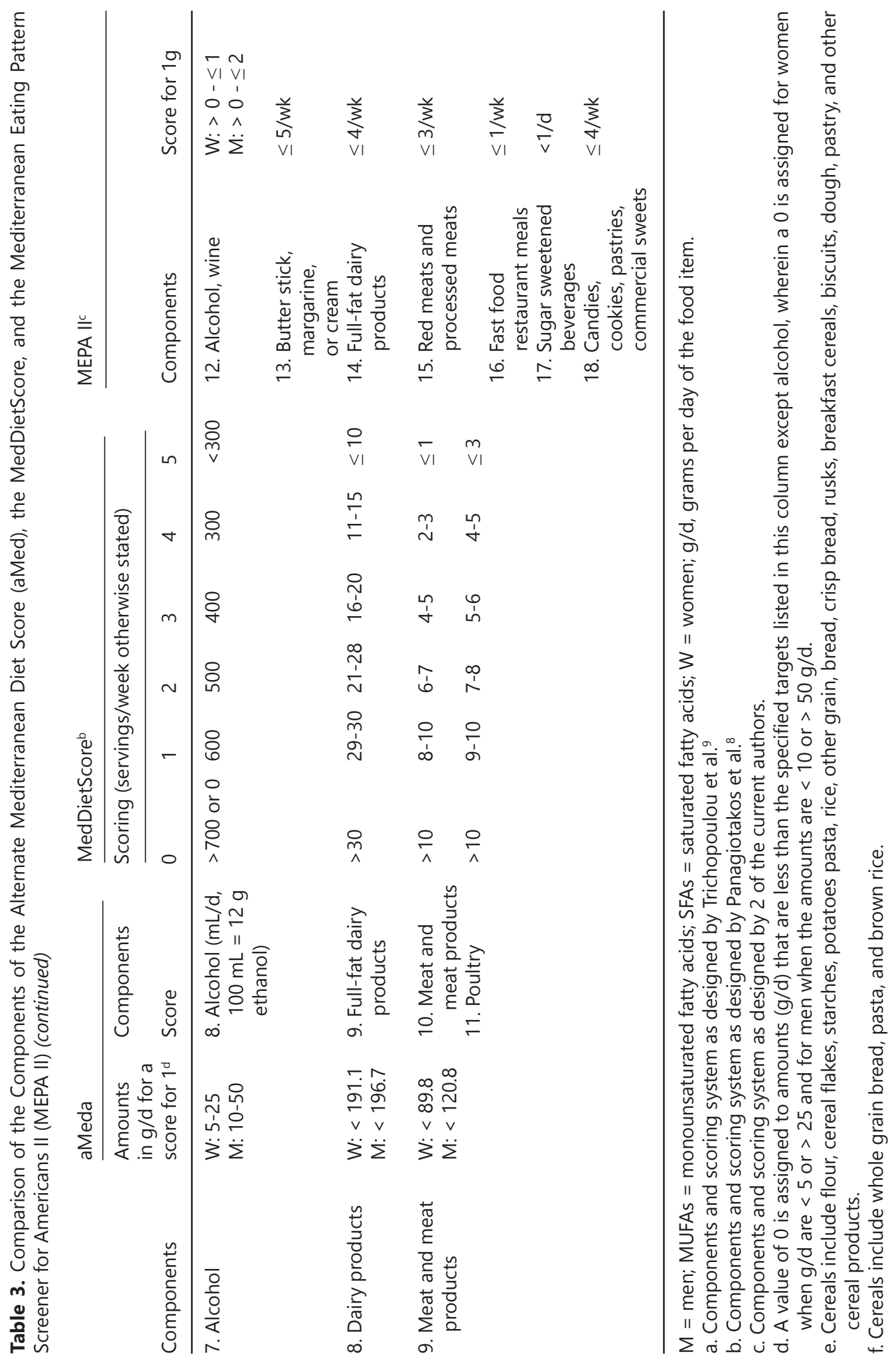


display the percentage of met food group targets as each food is entered ( https://www.supertracker.usda.gov/foodtracker.aspx ). However, none of the dietary patterns put forth by either the USDA/ Department of Health and Human Services or the Harvard researchers can be easily scored without the use of a nutrient and food composition database. Most nutrient database software applications will rely on the Food Pattern Equivalents Database, which is a tool in which complex food mixtures such as casseroles, build-your-own salads, and milkshakes are disaggregated and assigned to specific food groups so that all food components are included. ${ }^{26}$

Moreover, for several scoring algorithms (HEI 2010, DASH, AHEI 2010, and aMed), nutrient intakes must be quantified to determine whether intakes exceed the specified cutoff amount. Of note, in HEI 2010 (Table $2)$, nutrient analyses are necessary to compute scores for 1) polyunsaturated fatty acids plus monounsaturated fatty acids to saturated fatty acid ratios, 2) dietary sodium (mg), and 3) energy (kcal). Similar requirements exist for computing AHEI 2010, DASH, and aMed eating patterns (e.g., sodium, energy, fat, saturated fat, trans fat, and monounsaturated fat). Once again, the present authors are unaware of any brief, validated screeners to assess accordance to these dietary patterns in the literature for the American population.

To address these limitations and to afford more immediate feedback to clients wishing to adopt a more Mediterranean-style pattern, researchers developed a relatively simple screener known as MEPA II as shown in Table 3. MEPA II can be easily scored by the individual or the health professional to track healthy food behaviors that comprise a Mediterranean-like dietary pattern without the use of a food composition database. Although this screener does not replace the more complete dietary assessment method with the specific food compositional analysis, the MEPA II screener can be used to track individual food and beverage intakes qualitatively over time. MEPA II was modeled after the 14-point Mediterranean Diet Adherence Screener, ${ }^{23}$ which was used to measure accordance to the Mediterranean diet in Spanish adults at high risk of cardiovascular disease in the PREvencion con Dleta MEDiterranea trial. ${ }^{27}$ MEPA II incorporates much of the evidence our group and others have observed for specific foods and dietary patterns associated with reduced cognitive decline or incident Alzheimer disease ${ }^{28,29}$ but also includes additional modifications (convenience package foods, avocados, peanuts apart from other nuts, and so on). These were made so that the screener could be used in multiple outpatient clinics, especially for those with Parkinson disease, epilepsy, heart disease, and gastrointestinal disorders. 


\section{Importance of choosing a healthy dietary pattern}

Accordance to dietary patterns that align closely with the DGA or other recognized healthy dietary patterns have been associated with marked reductions in deaths attributable to diet-related chronic diseases. In a recent systematic review and meta-analysis of 15 different cohort studies (34 reports) of over 1 million participants, Schwingshackl and Hoffmann ${ }^{30}$ examined associations between 3 indices (HEI 2010, AHEI 2010, and DASH) and the risk of all-cause mortality and the incidence of and mortality from 4 major chronic diseases, cancer, cardiovascular disease, type 2 diabetes mellitus, and neurodegenerative diseases. High-quality diet patterns (HEI 2010, AHEI 2010, and DASH) were associated with a lower risk of all-cause and chronic disease deaths by as much as $15 \%$ to $22 \%$. These findings lend support to the value of adopting any of these healthy patterns for greater public health.

The scoring tool for a Mediterranean-like dietary pattern (aMed) was used in conjunction with 3 other tools/patterns (DASH, HEI-2010, and AHEl 2010) on the FFQ responses of 424,662 persons aged 50 to 71 years from the NIH-AARP Diet and Health Study. Associations between the dietary pattern scores and mortality or incident diseases were examined after 15 years of follow-up. ${ }^{31}$ Once again, accordance to each of these dietary patterns (aMed, DASH, HEI 2010, and AHEI 2010) was associated with protection against cardiovascular and cancer mortality. Because observed associations may differ across different cohorts, the Dietary Patterns Methods Project (DPMP) group included the NIH-AARP Diet and Health Study respondents in addition to those of 2 other large cohorts (Multiethnic Cohort and the Women's Health Initiative Observational Study) to examine accordance to 4 different dietary patterns in relation to all-cause mortality or mortality from cardiovascular disease and cancer. ${ }^{32}$ There was considerable consistency across these tools, with 20\% lower risk in mortality among those with high dietary quality scores. The congruence observed by the DPMP group and others ${ }^{31,33}$ lends further support to the value of evidence-based recommendations in the DGA.

\section{Conclusions}

Several healthy dietary patterns are advocated by the 2015 Dietary Guidelines for Americans Committee and other nutrition professionals. An appreciation of how dietary patterns are developed and operationalized is critical for all health care professionals. There is a plethora of 
scoring tools for these patterns, but few are brief and amenable to a clinic setting. The use of standardized scoring algorithms for dietary patterns shown to be associated with better health outcomes is a start for all health care professionals, including nurse practitioners. Knowledge of the evidence supporting the use of these patterns can foster greater acceptance of changes in the foods that individuals choose to eat and the healthfulness of the food environment.

In compliance with national ethical guidelines, the authors report no relationships with business or industry that would pose a conflict of interest.

\section{References}

1. United States Department of Agriculture. Scientific Report of the 2015 Dietary Guidelines Advisory Committee. Version current March 17, 2016. http://health. gov/dietaryguidelines/2015-scientific-report/ Accessed March 1, 2016.

2. United States Department of Agriculture, Department of Health and Human Services. Dietary Guidelines for Americans 2015-2020. http://health.gov/ dietaryguidelines/2015/guidelines/Accessed March 2016.

3. USDA. 2015 Dietary Guidelines Advisory Committee. Federal presentations. http://www.health.gov/dietaryguidelines/2015-binder/2015/historyCurrentUse. aspx Accessed March 1, 2016.

4. Wirfält AKE, Jeffery RW. Using cluster analysis to examine dietary patterns: nutrient intakes, gender, and weight status differ across food pattern clusters. J Am Diet Assoc. 1997;97:272-279.

5. Krebs-Smith SM, Subar AF, Reedy J. Examining dietary patterns in relation to chronic disease: matching measures and methods to questions of interest. Circulation. 2015;132:790-793.

6. Guenther PM, Casavale KO, Reedy J, et al. Update of the Healthy Eating Index: HEI-2010. J Acad Nutr Diet. 2013;113:569-580.

7. Guenther PM, Kirkpatrick SI, Reedy J, et al. The Healthy Eating Index-2010 is a valid and reliable measure of diet quality according to the 2010 dietary guidelines for Americans. J Nutr. 2014;144:399-407.

8. Panagiotakos DB, Pitsavos C, Arvaniti F, Stefanadis C. Adherence to the Mediterranean food pattern predicts the prevalence of hypertension, hypercholesterolemia, diabetes and obesity, among healthy adults; the accuracy of the MedDietScore. Prev Med. 2007;44:335-340.

9. Trichopoulou A, Bamia C, Trichopoulos D. Mediterranean diet and survival among patients with coronary heart disease in Greece. Arch Intern Med. 2005;165:929-935. 
10. Fung TT, McCullough ML, Newby PK, et al. Diet-quality scores and plasma concentrations of markers of inflammation and endothelial dysfunction. Am J Clin Nutr. 2005;82:163-173.

11. Appel LJ, Moore TJ, Obarzanek E, et al. A clinical trial of the effects of dietary patterns on blood pressure. DASH Collaborative Research Group. N Engl J Med. 1997;336:1117-1124.

12. Fung TT, Chiuve SE, McCullough ML, Rexrode KM, Logroscino G, Hu FB. Adherence to a DASH-style diet and risk of coronary heart disease and stroke in women. Arch Intern Med. 2008;168:713-720.

13. Sarkar D. Comparison of different DASH indices and prevalence of hypertension among a Midwestern sample of Hispanic adults. https://search. proquest.com/openview/e6ad3d198344e0413a810dae0f5617b4/1?pq-origsite $=$ gscholar $\& \mathrm{cbl}=18750 \&$ diss $=y$

14. Tangney CC. DASH and Mediterranean-type dietary patterns to maintain cognitive health. Curr Nutr Rep. 2014;3:51-61.

15. Lourida I, Soni M, Thompson Coon J, et al. Mediterranean diet, cognitive function, and dementia: a systematic review. Epidemiology. 2013;24: 479-489.

16. Newby PK, Muller D, Tucker KL. Associations of empirically derived eating patterns with plasma lipid biomarkers: a comparison of factor and cluster analysis methods. Am J Clin Nutr. 2004;80:759-767.

17. Hoffmann K, Schulze MB, Schienkiewitz A, Nöthlings U, Boeing H. Application of a new statistical method to derive dietary patterns in nutritional epidemiology. Am J Epidemiol. 2004;159:935-944.

18. Kennedy ET, Ohls J, Carlson S, Fleming K. The Healthy Eating Index: design and applications. J Am Diet Assoc. 1995;95:1103-1108.

19. Guenther PM, Reedy J, Krebs-Smith SM. Development of the Healthy Eating Index-2005. J Am Diet Assoc. 2008;108:1896-1901.

20. McCullough ML, Feskanich D, Stampfer MJ, et al. Diet quality and major chronic disease risk in men and women: moving toward improved dietary guidance. Am J Clin Nutr. 2002;76:1261-1271.

21. Chiuve SE, Fung TT, Rimm EB, et al. Alternative dietary indices both strongly predict risk of chronic disease. J Nutr. 2012;142:1009-1018.

22. Sacks FM, Svetkey LP, Vollmer WM, et al. Effects on blood pressure of reduced dietary sodium and the Dietary Approaches to Stop Hypertension (DASH) diet. DASH-Sodium Collaborative Research Group. N Engl J Med. 2001;344:3-10.

23. Blumenthal JA, Babyak MA, Hinderliter A, et al. Effects of the DASH diet alone and in combination with exercise and weight loss on blood pressure and cardiovascular biomarkers in men and women with high blood pressure: the ENCORE study. Arch Intern Med. 2010;170:126-135.

24. Lin PH, Appel LJ, Funk K, et al. The PREMIER intervention helps participants follow the Dietary Approaches to Stop Hypertension dietary pattern and the current Dietary Reference Intakes recommendations. J Am Diet Assoc. 2007; 107:1541-1551. 
25. Fung TT, Rexrode KM, Mantzoros CS, Manson JE, Willett WC, Hu FB.

Mediterranean diet and incidence of and mortality from coronary heart disease and stroke in women. Circulation. 2009;119:1093-1100.

26. ARS USDA. Food Patterns Equivalents Database. Version current. http://www. ars.usda.gov/Services/docs.htm?docid=23869 December 15, 2014. Accessed March 1, 2016.

27. Estruch R, Ros E, Salas-Salvado J, et al. Primary prevention of cardiovascular disease with a Mediterranean diet. N Engl J Med. 2013;368:1279-1290.

28. Morris MC, Tangney CC, Wang Y, et al. MIND diet slows cognitive decline with aging. Alzheimers Dement. 2015;11:1015-1022.

29. Morris MC, Tangney CC, Wang Y, Sacks FM, Bennett DA, Aggarwal NT. MIND diet associated with reduced incidence of Alzheimer's disease. Alzheimers Dement. 2015;11:1007-1014.

30. Schwingshackl L, Hoffmann G. Diet quality as assessed by the Healthy Eating Index, the Alternate Healthy Eating Index, the Dietary Approaches to Stop Hypertension score, and health outcomes: a systematic review and metaanalysis of cohort studies. J Acad Nutr Diet. 2015;115:780-800.e5.

31. Reedy J, Krebs-Smith SM, Miller PE, et al. Higher diet quality is associated with decreased risk of all-cause, cardiovascular disease, and cancer mortality among older adults. J Nutr. 2014;144:881-889.

32. Liese AD, Krebs-Smith SM, Subar AF, et al. The Dietary Patterns Methods Project: synthesis of findings across cohorts and relevance to dietary guidance. J Nutr. 2015;145:393-402.

33. Harmon BE, Boushey CJ, Shvetsov YB, et al. Associations of key diet-quality indexes with mortality in the multiethnic cohort: the dietary patterns methods project. Am J Clin Nutr. 2015;101:587-597. 